\title{
Hoarseness in an older adult: Ortner syndrome
}

\author{
Wei Ji Koh, Mawaddah Azman \\ Koh WJ, Azman M. Hoarseness in an older adult: Ortner syndrome. Malays Fam Physician. 2021;16(3);129-131. https://doi.org/10.51866/tyk1244
}

\section{Keywords:}

Voice disorders, dysphonia,

recurrent laryngeal nerve

injuries.

\section{Authors:}

\author{
Mawaddah Azman \\ (Corresponding author) \\ $M D, M S$ \\ Department of Otorhinolaryngology- \\ Head and Neck Surgery, 9th floor \\ Clinical Block, UKM Medical Centre \\ Faculty of Medicine, Jalan Yaacob \\ Latiff, Bandar Tun Razak \\ Kuala Lumpur, Malaysia \\ Email: mawaddah1504@yahoo.com
}

\section{Koh Wei Ji}

$M D$

Department of OtorhinolaryngologyHead and Neck Surgery, 9th floor

\section{Clinical Block, UKM Medical Centre}

Faculty of Medicine, Jalan Yaacob

Latiff, Bandar Tun Razak

Kuala Lumpur, Malaysia

\begin{abstract}
A detailed examination in an older adult presenting with chronic hoarseness is mandatory to exclude an upper aerodigestive tract malignancy. We describe a 71-year-old chronic smoker with essential hypertension who presented with hoarseness and vocal fatigue for 5 months. Laryngoscopic examination showed left vocal fold paralysis with phonation gap. No growth was seen at all laryngeal and hypopharyngeal subsites. The rest of the head and neck, chest, upper limbs and neurovascular examination were unremarkable. A plain chest radiograph demonstrated a cause for the left recurrent laryngeal nerve palsy. The diagnosis, complications and definitive management of the underlying cause and resultant voice problem will be discussed.
\end{abstract}

\section{Case summary}

A71-year-old man who was a chronic smoker with a background history of essential hypertension presented with hoarseness for 5 months. The symptom was sudden in onset and associated with vocal fatigue. The symptom was not worsening, and no aspiration symptoms were noted. There was no noisy breathing, dyspnoea, dysphagia or neck swelling. Laryngoscopic examination showed left vocal fold paralysis with phonation gap. No growth was seen at all laryngeal and hypopharyngeal subsites. The rest of the head and neck, chest, upper limbs and neurovascular examination were unremarkable. Baseline blood investigations, including a full blood count and a renal profile, were within the normal ranges. A plain chest radiograph was taken as part of the radiological investigation (Figure1).

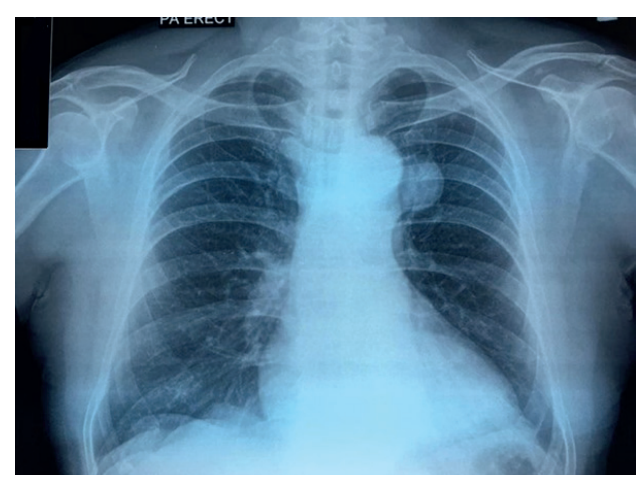

Figure 1. Plain chest radiograph

\section{Questions}

1) What abnormality is seen in the radiograph?

2) What differential diagnoses arise from an older adult presenting with chronic hoarseness?

3) What complication can potentially arise from the left vocal fold paralysis?

4) List the management for the above condition.

\section{Answers}

1) Description of the chest radiograph:

a. There is enlargement of the aortic knob and widening of the superior mediastinal silhouette. The heart and lungs otherwise appear normal.

2) Differential diagnoses for an older adult presenting with chronic hoarseness

a. Inflammation: chronic laryngitis, tuberculosis, syphilis

b. Tumours

i. Malignant: glottic carcinoma

ii. Benign: vocal nodule, vocal polyp, vocal cyst

c. Paralysis: unilateral recurrent laryngeal nerve paralysis secondary to iatrogenic cause (40\%), for example, thyroidectomy, neck surgery, thoracic surgery and spine surgery; idiopathic cause (25\%); malignant cause (25\%), for example, lung carcinoma, oesophageal carcinoma, metastatic lymphadenopathy, and other rare causes like Ortner syndrome (less than $5 \%)^{1}$ 
3) Complication

a. Aspiration pneumonitis secondary to non-compensated recurrent laryngeal nerve palsy ${ }^{1}$

4) Management

a. Medical management of thoracic aortic aneurysm

i. Smoking cessation.

ii. Good control of hypertension to aim for blood pressure of $\leq 120 / 80 \mathrm{mmHg}^{2}$

iii. Beta-blockers like propranolol and metoprolol have been shown to slow the rate of growth by reducing the stress to the aortic wall. ${ }^{2}$ Losartan, an angiotensin-receptor blocker, interferes with the signalling of the TGF-beta protein and slows the growth of aortic aneurysms in Marfan syndrome. ${ }^{3}$

iv. Serial follow-up CT angiogram to assess the progression and size of the aneurysm in patients managed conservatively.

b. Surgical management of thoracic aortic aneurysm

i. Open repair of aneurysm is considered in symptomatic patients or aneurysm size of $5 \mathrm{~cm}$ or more in asymptomatic patients. ${ }^{2}$

ii. Thoracic endovascular aortic repair (TEVAR) is less invasive, less expensive and carries a lower operative risk compared to standard open repair of aneurysm, especially if the patient is at high risk for open intervention. ${ }^{1,4}$

c. Management of recurrent laryngeal nerve palsy

i. Voice improvement is expected within weeks or months following successful surgical intervention for the thoracic aneurysm. Hence, office injection laryngoplasty with hyaluronic acid can be performed by the laryngologist to alleviate hoarseness while waiting for definitive surgical intervention. ${ }^{5}$

ii. Permanent medialisation thyroplasty under local anaesthesia if surgical intervention is not considered or poor anaesthesia candidate. ${ }^{1}$

\section{Acknowledgements}

This work did not receive funding from any governmental or non-governmental organizations. The institutional Ethical Review Board of UKM approved the conduct of this work with approval ID JEP-2020-128.

\section{Conflicts of interest}

All authors declare no conflicting interests.

How does this paper make a difference to general practice?

- Hoarseness in a patient is encountered regularly in daily general practice, and its subsequent management can often be elusive.

- This paper highlights the importance of considering unilateral vocal fold paralysis in an older adult who was otherwise asymptomatic of an aortic aneurysm, despite having the risk factor of essential hypertension.

- A detailed and early ear, nose and throat examination in an older adult with risk factors presenting with hoarseness is mandatory to exclude an upper aerodigestive tract malignancy.

- A simple chest radiograph is useful in further management of such patients to confirm the diagnosis of Ortner syndrome or exclude aspiration pneumonitis and lung cancer.

- In addition to avoiding unnecessary invasive diagnostic procedures, a prompt diagnosis provides patients with reassurance and prevents unnecessary anxiety over the possibility of malignancy. 


\section{References}

1. Dayangku NPS, Marina MB, Mawaddah A, Sharifa Ezat WP, Abdullah S. Gore-Tex medialisation thyroplasty for unilateral vocal cord palsy: a tertiary centre 7 years experience. IIUM Med J Malaysia. 2016 Nov26;15(2).

2. Salameh MJ, Black JH 3rd, Ratchford EV. Thoracic aortic aneurysm. Vasc Med. 2018Dec[12 May 2021];23(6):573-8. doi:10.1177/1358863X18807760.
3 Brooke, ES, Habashi, JP, Judge, DP. Angiotensin II blockade and aortic-root dilationin Marfan's syndrome. $N$ Engl J Med. 2008;358:2787-95.

4. Ismazizi Z, Zainal AA. Thoracic aortic aneurysm as a cause of Ortner's syndrome -a case series. Med J Malaysia. 2016 Jun;71(3):139-41. PMID:27495889.
5. Salinas JB, Chhetri DK. Injection laryngoplasty: techniques and choices of fillers. Curr Otorhinolaryngol Rep. 2014;2:131-6. 